\title{
Collision-Induced Spin Flip of Hydrogen Atoms
}

\author{
OLDWIG VON ROOS \\ Jet Propulsion Laboratory, California Institute of Technology, Pasadena, California \\ (Received March 30, 1959)
}

\begin{abstract}
The cross section of a radiationless electronic spin-state transition (triplet-singlet transition) of two colliding, unexcited, hydrogen atoms has been calculated for low relative velocities. The low-velocity limit of this cross section exhibits a logarithmic divergence and decreases rapidly to a value of the order of $\pi r_{0}{ }^{2}$ $\left(r_{0}=\right.$ classical electron-radius $\left.e^{2} / 4 \pi m c^{2}\right)$ with increasing energy of relative motion.
\end{abstract}

\section{INTRODUCTION}

$I^{\mathrm{N}}$ $\mathrm{N}$ this paper we investigate a collision-induced nonradiative triplet-singlet transition of two hydrogen atoms at low impact velocities. The transition is caused by the spin interaction between the systems, an interaction which is ultimately due to an exchange of transverse photons between the various particles (see Sec. 2). This transition, which can be realized experimentally by crossed beams of polarized hydrogen atoms, is interesting from the point of view of resonance collisions. It is well known ${ }^{1}$ that resonance collisions are rearrangement collisions in which the internal energy of the colliding systems is redistributed without causing any change in the kinetic energy of the relative motion. Usually the transition probability of such a reaction is directly proportional to the time the colliding systems spend within each other's interaction sphere, so that the corresponding cross section behaves as $v^{-1}$ even for very small velocities, $v$, of the relative motion. The specific reaction we are considering here has some similarity to a resonance collision inasmuch as the state vector of the system undergoes a transition without any change of the kinetic energy. On the other hand, it also has some similarity to an ordinary elastic scattering since the state of each hydrogen atom separately is not altered. It is only the symmetry of the wave function of the combined system which is changed, the electrons staying in the ground state throughout. It is therefore interesting to note, as we are going to show in part 3, that at low velocities the cross section of the spin-flip transition considered here tends logarithmically to infinity. This behavior is exactly intermediate between that of a true resonance collision $\left(\sim v^{-1}\right)$ and that of an elastic scattering, the latter tending to a finite limit as $v$ approaches zero.

However, except for the logarithmic divergence of the cross section which is more or less of academic interest since it makes itself felt only at extremely small relative velocities (temperature equivalent $<0.1^{\circ} \mathrm{K}$ ), the spin-flip cross section is rather small. The probability of a spin flip per encounter, given by the ratio of the spin-flip cross section to the elastic scattering cross section, turns out to have the value $10^{-10}$ for velocities greater than $1^{\circ} \mathrm{K}$ temperature equivalent.

${ }^{1}$ H. S. W. Massey and E. H. S. Burhop, Electronic and Ionic Impact Phenomena (Clarendon Press, Oxford, 1956), p. 442.
In Sec. 2 of this paper we shall derive a general formula for any radiationless spin-dependent transition involving an arbitrary number of fermions. In Sec. 3 we shall apply the result of Sec. 2 to the specific problem at hand, namely the scattering of two polarized unexcited hydrogen atoms accompanied by spin flip of one of the electrons.

\section{NONRADIATIVE TRANSITIONS OF A SYSTEM OF FERMIONS DUE TO SPIN INTERACTIONS}

The spin interaction of charged fermions is usually obtained from semiclassical considerations ${ }^{2}$ or from the Breit equation. ${ }^{3}$ We wish to give here a brief account of radiationless transitions due to this interaction. We start with the Schrödinger equation of a set of FermiDirac particles coupled to the quantized transverse electromagnetic field. The equation of motion is

$$
\left(\frac{\hbar}{i} \frac{\partial}{\partial t}+H_{C}+H_{P}+H_{\text {tr }}+H_{\text {inter }}\right) \phi=0 .
$$

Here $H_{C}$ is the Coulomb interaction of the particles, $H_{P}$ is the particle Hamiltonian (see below), $H_{\mathrm{tr}}$ is the energy of the free photons, and $H_{\text {inter }}$ finally is the interaction energy between the particles and the photon field :

$$
H_{\text {inter }}=e \sum_{n} \boldsymbol{\alpha}_{n} \cdot \mathbf{A}\left(\mathbf{r}_{n}\right),
$$

where $\mathbf{A}(\mathbf{r})$ represents the quantized vector potential and $\boldsymbol{\alpha}_{n}$ are the usual spin matrices of the $n$th particle. The particle Hamiltonian is given by

$$
H_{P}=\sum_{n}\left\{m_{n} c^{2} \boldsymbol{\beta}_{n}+\boldsymbol{\alpha}_{n} \cdot\left(\frac{\hbar c}{i} \nabla_{n}+e \mathbf{A}^{\mathrm{ex}}\left(\mathbf{r}_{n}\right)\right)\right\},
$$

where the sum extends over all particles with masses $m_{n}$ and $\mathbf{A}^{\mathrm{ex}}$ is a prescribed external field. The vector potential $\mathbf{A}$ in turn is given by

$\mathbf{A}(\mathbf{r})=\left(\frac{\hbar c}{2(2 \pi)^{3}}\right)^{\frac{1}{2}} \int \frac{d^{3} k}{\sqrt{ } k} \sum_{r} \mathbf{e}_{k}{ }^{(r)}\left(a_{k}{ }^{(r)}+a_{-k}{ }^{(r) \dagger}\right) e^{i \mathbf{k} \cdot \mathbf{r}}$.

Here $\mathbf{e}_{k}^{(r)}$ are unit vectors in direction of polarization,

${ }^{2}$ H. A. Kramers, Quantum Mechanics (North-Holland Publishing Company, Amsterdam, 1957).

${ }^{3}$ H. A. Bethe and E. E. Salpeter, Encyclopedia of Physics (Springer-Verlag, Berlin, 1957), Vol. 35, p. 256 ff. 
$\mathbf{k}$ is the wave vector $\left(\mathbf{k} \cdot \mathbf{e}_{k}{ }^{(r)}=0\right)$ and $a_{k}{ }^{(r)}, a_{k}{ }^{(r) \dagger}$ are the usual annihilation and creation operators of the photon field which satisfy

$$
\left[a_{k}{ }^{(r)}, a_{k^{\prime}}\left(r^{\prime}\right) \dagger\right]=\delta_{r r^{\prime}} \delta\left(k-k^{\prime}\right) .
$$

All other commutators vanish.

We solve Eq. (1) with the following "ansatz":

$$
\begin{array}{r}
\phi=\sum_{N, r} \int d^{3} k_{1} \cdots d^{3} k_{N} \varphi_{N}\left(\mathbf{r}_{1} \cdots\right. \\
\left.\mathbf{r}_{n}, t ; \mathbf{k}_{1} r_{1} \cdots \mathbf{k}_{N} r_{N}\right) \\
\times \Omega_{N}\left(\mathbf{k}_{1} r_{1} \cdots \mathbf{k}_{N} r_{N}\right),
\end{array}
$$

where $\Omega_{N}$ is eigenvector of $H_{\mathrm{tr}}$,

$H_{\mathrm{tr}} \Omega_{N}\left(\mathbf{k}_{1} r_{1} \cdots \mathbf{k}_{N} r_{N}\right)=\hbar c \sum_{n=1}^{N}\left|\mathbf{k}_{n}\right| \Omega_{N}\left(\mathbf{k}_{1} r_{1} \cdots \mathbf{k}_{N} r_{N}\right)$.

Inserting Eq. (6) into Eq. (1) and eliminating the $\Omega_{N}$ gives a system of coupled integro-differential equations for the amplitudes $\varphi_{N}$. Truncating the series (6) for $N=N^{\prime}$ leaves us with a set of $N^{\prime}$ coupled equations. This is the well-known $N^{\prime}$ th Tamm-Dancoff approximation.

Let us define the complete set of orthogonal functions $\psi_{m}$ by

$$
\left(\frac{\hbar}{i} \frac{\partial}{\partial t}+H_{C}+H_{P}\right) \psi_{m}=0
$$

so that

$$
\psi_{m}=e^{-(i t / \hbar) E_{m}} \bar{\psi}_{m}\left(\mathbf{r}_{1} \cdots \mathbf{r}_{n}\right)
$$

with

$$
\left(-E_{m}+H_{C}+H_{P}\right) \bar{\psi}_{m}=0 .
$$

We now impose the following initial conditions on the amplitudes $\varphi_{N}$ of expansion (6) in the remote past:

$$
\begin{aligned}
\varphi_{N}\left(\mathbf{r}_{1} \cdots \mathbf{r}_{n},-\infty ; \mathbf{k}_{1} \boldsymbol{r}_{1} \cdots \mathbf{k}_{N} \boldsymbol{r}_{N}\right) \\
\quad=\delta_{N N} \psi_{m} \delta\left(\mathbf{k}_{1}-\mathbf{k}_{1}{ }^{\prime}\right) \cdots \delta\left(\mathbf{k}_{N}-\mathbf{k}_{N}{ }^{\prime}\right) .
\end{aligned}
$$

The meaning of Eq. (11) is obviously that the system of particles is in a definite state as given by (8) and that furthermore there are $N^{\prime}$ photons with specified momenta $\mathbf{k}_{n}{ }^{\prime}$.

The probability then that at time $t$ the system is in the state $\psi_{m}$. $\Omega_{N^{\prime \prime}}$ with specified photon momenta and polarization is obtained from the square of the following matrix element:

$$
\left\langle\psi_{m^{\prime}} \mid \varphi_{N^{\prime \prime}}\left(\mathbf{r}_{1} \cdots \mathbf{r}_{n}, t ; \mathbf{k}_{1} r_{1} \cdots \mathbf{k}_{N^{\prime \prime}} \boldsymbol{r}_{N^{\prime \prime}}\right)\right\rangle,
$$

from which the corresponding transition probability may be obtained by taking the limit $t=+\infty$ (see below). Being interested in nonradiative transitions we assume that initially no photons are present:

$$
\left.\begin{array}{ll}
\varphi_{N}=0 & \text { for } N \neq 0 \\
\varphi_{0}=\psi_{m} & \text { for } N=0
\end{array}\right\} \text { for } t=-\infty .
$$

We assume further that two-photon amplitudes may already be neglected (first Tamm-Dancoff approxima- tion). The expansion (6) reads in this case

$\phi=\varphi_{0}\left(\mathbf{r}_{1} \cdots \mathbf{r}_{n}, t\right) \Omega_{0}$

$$
+\sum_{r} \int d^{3} k \varphi_{1}\left(\mathbf{r}_{1} \cdots \mathbf{r}_{n}, t ; k\right) \Omega_{1}(k, r) .
$$

Inserting this expression into Eq. (1) and observing Eqs. (4) and (5) gives the following set of equations for the amplitudes $\varphi_{0}$ and $\varphi_{1}$ :

$$
\begin{gathered}
\left(\frac{\hbar}{i} \frac{\partial}{\partial t}+H_{C}+H_{P}+\hbar c k\right) \varphi_{1}=-e\left(\frac{\hbar c}{2}\right)^{\frac{1}{2}} D_{-}{ }^{(r)}(k) \varphi_{0}, \\
\left(\frac{\hbar}{i} \frac{\partial}{\partial t}+H_{C}+H_{P}\right) \varphi_{0} \\
=-e\left(\frac{h c}{2}\right)^{\frac{1}{2}} \sum_{r} \int d^{3} k D_{+}{ }^{(r)}(k) \varphi_{1},
\end{gathered}
$$

with

$$
D_{ \pm}^{(r)}(k)=(2 \pi)^{-\frac{3}{2}} k^{-\frac{1}{2}} \sum_{n} \boldsymbol{\alpha}_{n} \cdot \mathbf{e}_{k}{ }^{(r)} e^{ \pm i \mathbf{k} \cdot \mathbf{r}_{n}}
$$

The system (14) is to be solved together with the initial conditions

$$
\begin{gathered}
\varphi_{1}\left(\mathbf{r}_{1} \cdots \mathbf{r}_{n},-\infty ; k, r\right)=0 \\
\varphi_{0}\left(\mathbf{r}_{1} \cdots \mathbf{r}_{N},-\infty\right)=\bar{\psi}_{m} .
\end{gathered}
$$

Expanding $\varphi_{1}$ with respect to the set $\bar{\psi}_{m}[$ Eq. (10)], we have

$$
\varphi_{1}=\sum_{n} b_{n} \bar{\psi}_{n}
$$

$$
\left(\frac{\hbar}{i} \frac{\partial}{\partial t}+E_{n}+\hbar c k\right) b_{n}=-e\left(\frac{\hbar c}{2}\right)^{\frac{1}{2}}\left\langle\bar{\psi}_{n}\left|D_{-}{ }^{(r)}(k)\right| \varphi_{0}\right\rangle .
$$

The initial condition (16) for $\varphi_{1}$ is satisfied with the following solution of Eq. (18) :

$$
\begin{aligned}
b_{n}=-\lim _{\epsilon=0} \frac{e}{h}\left(\frac{\hbar c}{2}\right)^{\frac{1}{2}} \int_{-\infty}^{+\infty} d l \int_{-\infty}^{+\infty} & d t^{\prime} \frac{e^{i l\left(t-t^{\prime}\right)}}{l+E_{n} / \hbar+c k-i \epsilon} \\
& \times\left\langle\bar{\psi}_{n}\left|D_{-}{ }^{(r)}\right| \varphi_{c}{ }^{\prime}\right\rangle
\end{aligned}
$$

where the prime on $\varphi_{0}^{\prime}$ indicates that it contains $t^{\prime}$ as an argument. $\varphi_{0}$ is, to first order,

$$
\varphi_{0}=\bar{\psi}_{m} e^{-i t E_{m} / \hbar}
$$

according to Eqs. (14) and (16). Inserting Eq. (20) into (19) and performing the indicated integrations yields

$$
\begin{aligned}
b_{n}=-\frac{e}{h}\left(\frac{\hbar c}{2}\right)^{\frac{1}{2}}\left\langle\bar{\psi}_{m}\right| D_{-}{ }^{(r)} & \left.(k) \bar{\psi}_{m}\right\rangle \\
& \times \frac{e^{-i t E_{m} / \hbar}}{\hbar^{-1}\left(E_{n}-E_{m}\right)+c k-i \epsilon},
\end{aligned}
$$


from which it follows that $\varphi_{1}$ is simply given by

$$
\begin{aligned}
\varphi_{1} & =\sum_{n} b_{n} \bar{\psi}_{n} \\
& =-\frac{e}{h}\left(\frac{\hbar c}{2}\right)^{\frac{1}{2}}\left[\left(H-E_{m}\right) / \hbar+c k+i \epsilon\right]^{-1} D_{-}{ }^{(r)}(k) \psi_{m},
\end{aligned}
$$

with $H=H_{C}+H_{P}$.

Equation (14b) now gives

$$
\begin{aligned}
\left(\frac{\hbar}{i} \frac{\partial}{\partial t}+H\right) & \varphi_{0}=\frac{e^{2} c}{4 \pi} \sum_{r} \int d^{3} k D_{+}{ }^{(r)}(k) \\
& \times\left[\left(H-E_{m}\right) / \hbar+c k+i \epsilon\right]^{-1} D_{-}{ }^{(r)}(k) \psi_{m} .
\end{aligned}
$$

Expanding again

$$
\varphi_{0}=\sum_{l} a_{l} \bar{\psi}_{l}
$$

we have from (23)

$$
\begin{aligned}
\left(\frac{\hbar}{i} \frac{\partial}{\partial t}+E_{l}\right) a_{l} & \\
=\frac{e^{2} c}{4 \pi} \sum_{r} \int d^{3} k\left\langle\bar{\psi}_{l}\right| D_{+}{ }^{(r)}(k)[(H & \left.\left.-E_{m}\right) / \hbar+c k\right]^{-1} \\
& \times D_{-}{ }^{(r)}(k)\left|\psi_{m}\right\rangle,
\end{aligned}
$$

with the initial condition (from 15) $a_{l}(-\infty)=\delta_{l m}$ so that analogous to Eq. (19) for $l \neq m$,

$$
\begin{aligned}
a_{l}= & \lim _{\epsilon=0} \frac{e^{2} c}{4 \pi \hbar} \int_{-\infty}^{+\infty} d l \int_{-\infty}^{+\infty} d t^{\prime} \frac{e^{i l\left(t-t^{\prime}\right)}}{l+\hbar^{-1} E_{l}-i \epsilon} \\
& \times \sum_{r} \int_{-\infty}^{+\infty} d^{3} k\left\langle\bar{\psi}_{l}\right| D_{+}{ }^{(r)}(k) \\
& \times\left[\left(H-E_{m}\right) / \hbar+c k\right]^{-1} D_{-}{ }^{(r)}(k)\left|\psi_{m}{ }^{\prime}\right\rangle .
\end{aligned}
$$

Performing the integration both over $l$ and $t^{\prime}$ in the last expression and multiplying by $e^{(i t / \hbar) / E_{l}}$ we obtain, for the transition amplitude of a radiationless transition from state $\psi_{m}$ to state $\psi_{l}$ in the limit $t=+\infty$,

$$
\begin{array}{r}
A_{l m}=a_{l} e^{i t E_{l} l \hbar}=\frac{e^{2} c}{4 \pi \hbar} \sum_{r} \int d^{3} k\left\langle\bar{\psi}_{l}\right| D_{+}{ }^{(r)}(k) \\
\left.\times\left[\left(H-E_{m}\right) / \hbar+c k\right]^{-1} D_{-}{ }^{(r)}(k) \bar{\psi}_{m}\right\rangle \\
\times \lim _{t=+\infty, \epsilon=0} \frac{e^{i t\left(E_{l}-E_{m}\right) / \hbar}}{\left(E_{l}-E_{m}\right) / \hbar-i \epsilon} .
\end{array}
$$

The limit which occurs in $(27)$ is given by ${ }^{4}$

$$
\lim _{t=+\infty} \lim _{\epsilon=+0} \frac{e^{i \alpha t}}{\alpha-i \epsilon}=2 \pi i \delta(\alpha) .
$$

Further reduction of the matrix element (27) is easily

${ }^{4}$ J. M. Jauch and F. Rohrlich, The Theory of Photons and Electrons (Addison-Wesley Press, Cambridge, 1955), p. 464. done, noting that $c k \gg\left(H-E_{m}\right) / \hbar$ at least for low $Z .^{5}$ Together with the defining equation (14c) for $D_{ \pm}$, the integration over $k$ yields

with $^{6}$

$$
A_{l m}=\frac{i}{\hbar}\left\langle\bar{\psi}_{l}\left|\sum_{n<n^{\prime}} B_{n n^{\prime}}\right| \bar{\psi}_{n}\right\rangle \delta\left(\frac{E_{l}-E_{m}}{\hbar}\right),
$$

$$
B_{n n^{\prime}}=\frac{e^{2}}{8 \pi r_{n n^{\prime}}}\left(\boldsymbol{\alpha}_{n} \cdot \boldsymbol{\alpha}_{n^{\prime}}+\frac{\boldsymbol{\alpha}_{n} \cdot \mathbf{r}_{n n^{\prime}} \boldsymbol{\alpha}_{n^{\prime}} \cdot \mathbf{r}_{n n^{\prime}}}{r_{n n^{\prime}}{ }^{2}}\right),
$$

the Breit operator and $\mathbf{r}_{n n^{\prime}}=\mathbf{r}_{n}-\mathbf{r}_{n^{\prime}}$ the relative distance between the particles $n$ and $n^{\prime}$. The transition probability follows from (28) in a standard manner ${ }^{7}$ and is given by

$$
P_{l m}=\frac{2 \pi}{\hbar}\left|\left\langle\bar{\psi}_{l}\left|\sum_{n<n^{\prime}} B_{n n^{\prime}}\right| \bar{\psi}_{m}\right\rangle\right|^{2} \delta\left(E_{m}-E_{l}\right) .
$$

\section{CALCULATION OF THE CROSS SECTION FOR SPIN FLIP OF TWO HYDROGEN ATOMS}

The particular reaction for which we wish to apply Eq. (30) is one in which two hydrogen atoms with aligned electronic spins are scattered by each other and simultaneously the spin of one of the electrons is reversed. More specifically, it is an elastic scattering of two unexcited $\mathrm{H}$ atoms accompanied by a tripletsinglet transition of the electronic state. Because of the smallness of the nuclear magnetic moment we shall assume the nuclei to be unpolarized. Since we are interested in low velocity encounters, we shall use the Pauli approximation for the matrix element $\left\langle\bar{\psi}_{l}\left|B_{n n^{\prime}}\right| \bar{\psi}_{m}\right\rangle$ responsible for the transition. The passage to the nonrelativistic limit is amply described in reference 3 . Particularly the following expression is obtained for the Breit operator in this approximation:

where

$$
B_{n n^{\prime}}=\frac{e^{2}}{4 \pi\left(2 m_{n} m_{n^{\prime}} c^{2}\right)}\left(V_{1}+V_{2}+V_{3}\right)
$$

$$
V_{1}=\frac{1}{r_{n n^{\prime}}}\left(\mathbf{p}_{n} \cdot \mathbf{p}_{n^{\prime}}+\frac{\mathbf{p}_{n} \cdot \mathbf{r}_{n n^{\prime}} \mathbf{p}_{n^{\prime}} \cdot \mathbf{r}_{n n^{\prime}}}{r_{n n^{\prime}}{ }^{2}}\right)
$$

is the orbit-orbit interaction,

$$
V_{2}=\frac{\hbar}{r_{n n^{\prime}}{ }^{3}}\left(\mathbf{r}_{n n^{\prime}} \times \mathbf{p}_{n}\right) \cdot \mathbf{\sigma}_{n^{\prime}}-\frac{\hbar}{r_{n n^{\prime}}{ }^{3}}\left(\mathbf{r}_{n n^{\prime}} \times \mathbf{p}_{n^{\prime}}\right) \cdot \mathbf{\sigma}_{n}
$$

the spin-orbit interaction, and

$$
\begin{aligned}
V_{3}=\hbar^{2} \boldsymbol{\sigma}_{n} \cdot \boldsymbol{\sigma}_{n^{\prime}}\left(\frac{4 \pi}{3} \delta^{3}\left(\mathbf{r}_{n n^{\prime}}\right)\right. & \left.-\frac{1}{2 r_{n n^{\prime}}{ }^{3}}\right) \\
& +\frac{3 \hbar^{2}}{2} \frac{\boldsymbol{\sigma}_{n} \cdot \mathbf{r}_{n n^{\prime}} \boldsymbol{\sigma}_{n^{\prime}} \cdot \mathbf{r}_{n n^{\prime}}}{r_{n n^{\prime}}}
\end{aligned}
$$

${ }^{5}$ Reference 3, p. 258.

${ }^{6}$ The factor $1 / 4 \pi$ is due to the choice of Heaviside units.

${ }^{7}$ Reference 4, p. 164. 
the spin-spin interaction, with $\mathbf{p}=(\hbar / i) \nabla$, and

$$
\boldsymbol{\sigma}=\left(\sigma_{x}, \sigma_{y}, \sigma_{z}\right)
$$

the usual Pauli spin matrices. The operator (31) acts on two-component Pauli spinors which in turn satisfy the nonrelativistic counterpart of Eq. (8).

Denoting the two protons of our system by $A$ and $B$ and the two electrons by 1 and 2 , we have six different interaction operators $B_{n n^{\prime}}$ corresponding to the possibilities $B_{A B}, B_{A 1}, B_{A 2}, B_{B 1}, B_{B 2}$, and $B_{12}$. We neglect $B_{A B}$ at once since it is smaller than $B_{A 1}$, etc. by the ratio $m / M$, where $m$ is the mass of an electron and $M$ the mass of a proton. Before proceeding with the actual evaluation of the matrix element, the wave functions of both the initial and the final state have to be obtained.

\section{a. The Wave Functions}

The equation satisfied by the wave functions is the ordinary Schrödinger equation ${ }^{8}$ for a two-proton, twoelectron system $\left(\mathbf{A}^{\mathrm{ex}}=0\right)$ :

$$
\begin{aligned}
{\left[-\left(\hbar^{2} / 2 M\right)\left(\nabla_{A}{ }^{2}+\nabla_{B}^{2}\right)-\left(\hbar^{2} / 2 m\right)\right.} \\
\left.\times\left(\nabla_{1}^{2}+\nabla_{2}^{2}\right)+H_{C}\right] \bar{\psi}=E \bar{\psi} .
\end{aligned}
$$

For the following calculations we use a spherical coordinate system with its $z$ axis along the vector $\mathbf{R}=\mathbf{r}_{A}-\mathbf{r}_{B}$, the relative distance between the nuclei. Furthermore we denote the spin functions by $S(1,2)$ and $S(A, B)$ for the electrons and nuclei, respectively. Defining $\alpha$ and $\beta$ by $\sigma_{z} \alpha=\alpha$ and $\sigma_{z} \beta=-\beta$ as usual, we have four possible configurations for each $S$. They are

$$
\left.\begin{array}{l}
S_{1}^{1}(1,2)=(1 / \sqrt{2})\left(\alpha_{1} \beta_{2}-\beta_{1} \alpha_{2}\right) \text { singlet, } \\
S_{1}{ }^{3}(1,2)=\alpha_{1} \alpha_{2} \\
S_{2}{ }^{3}(1,2)=\beta_{1} \beta_{2} \\
S_{3}{ }^{3}(1,2)=(1 / \sqrt{2})\left(\alpha_{1} \beta_{2}+\beta_{1} \alpha_{2}\right)
\end{array}\right\} \text { triplet, }
$$

and corresponding expressions for the nuclear spin functions $S(A, B)$.

The initial wave function may then be written as

$$
\begin{aligned}
\bar{\psi}_{m}=\chi_{n}{ }^{(A)}\left(\mathbf{r}_{A}, \mathbf{r}_{B}\right) S_{m}{ }^{n} & (A, B) \\
& \times \psi_{A}\left(\mathbf{r}_{1}, \mathbf{r}_{2} ; \mathbf{r}_{A}, \mathbf{r}_{B}\right) S_{m^{\prime}}{ }^{3}(1,2) .
\end{aligned}
$$

Here $\psi_{A}$ is the antisymmetric electronic wave function ${ }^{9}$ whereas $\chi_{n}{ }^{(A)}$ is the nuclear wave function which is symmetric if $n=3$ and antisymmetric if $n=1 . \chi_{n}{ }^{(A)}$ satisfies in the center-of-mass system

$$
\left[\nabla_{R^{2}}-\left(M / \hbar^{2}\right) v_{A}(R)+k^{2}\right] \chi_{n}{ }^{(A)}=0,
$$

where $v_{A}(R)$ is the repulsive interaction potential between two unexcited $\mathrm{H}$ atoms and $k^{2}=M E / \hbar^{2}$. According to (33) and (34) there are $3 \times 4=12$ possible initial states, 4 for each electron spin state $S_{m}^{3}(1,2)$.

\footnotetext{
${ }^{8}$ Neglecting a small spin-dependent contribution which can be shown to have no effect on the transition considered.

${ }^{9} \psi_{A}$ is also antisymmetric in the nuclear coordinates $\mathbf{r}_{A}$ and $\mathbf{r}_{B}$.
}

The final state may be represented by

$\bar{\psi}_{l}=\chi_{n}{ }^{(S)}\left(\mathbf{r}_{A}, \mathbf{r}_{B}\right) S_{m}^{n}(A, B)$

$$
\times \psi_{S}\left(\mathbf{r}_{1}, \mathbf{r}_{2} ; \mathbf{r}_{A}, \mathbf{r}_{B}\right) S_{1}^{1}(1,2),
$$

where $\psi_{S}$ is the symmetric electronic wave function and $\chi_{n}{ }^{(S)}$ the nuclear wave function satisfies (again in the center-of-mass system)

$$
\left[\nabla_{R^{2}}-\left(M / \hbar^{2}\right) v_{S}(R)+k^{2}\right] \chi_{n}^{(S)}=0,
$$

with $v_{S}(R)$ the attractive interaction potential between two unexcited $\mathrm{H}$ atoms. $\chi_{n}{ }^{(S)}$ is symmetric if $n=1$ and antisymmetric if $n=3$. Its symmetry behavior is opposite to that of $\chi_{n}{ }^{(A)}$ since the electronic wave function $\psi_{S}$ has the opposite symmetry of $\psi_{A}$ with respect to the nuclear coordinates $\mathbf{r}_{A}$ and $\mathbf{r}_{B}$. Both $\chi_{n}{ }^{(S)}$ and $\chi_{n}{ }^{(A)}$ belong to the continuum of scattering states. There are four possible final state wave functions (37) corresponding to the four possible nuclear spin states $S_{m}{ }^{n}(A, B)$.

For the electronic wave functions we used simple Heitler-London type orbitals. More specifically, we assumed for $\psi_{S}$ and $\psi_{A}$ (in atomic units)

$$
\left.\begin{array}{l}
\psi_{S} \\
\psi_{A}
\end{array}\right\}=\left(2 \pi^{2} N^{ \pm}\right)^{-\frac{1}{2}}\left(e^{-\left(r_{A 1}+r_{B 2}\right)} \pm e^{-\left(r_{A 2}+r_{B 1}\right)}\right)
$$

with $N^{ \pm}=1 \pm\left(1+R+R^{2}\right) e^{-2 R}$, the normalization factor. Wang $^{10}$ showed, using hydrogen-like orbitals $e^{-\alpha r}$ with adjustable screening parameter $\alpha$, that the effective nuclear charge $Z$ is not very different from unity. The wave function (38) is therefore sufficiently accurate for our purpose.

For the nuclear wave functions satisfying Eqs. (35) and (37), respectively, we made the following approximations which are entirely satisfactory for our purpose. The repulsive interaction potential $v_{A}(R)$ for the initial state has been replaced by a hard core,

$$
\begin{aligned}
v_{A}(R) & =0 & & \text { for } R>2 \\
& =\infty & & \text { for } R \leqslant 2 .
\end{aligned}
$$

Similarly for the final state,

$$
\begin{aligned}
v_{S}(R) & =0 & & \text { for } R>0.6 \\
& =\infty & & \text { for } R \leqslant 0.6 .
\end{aligned}
$$

Figure 1 shows the actual interaction potentials together with the approximations (39). The wave function $\chi_{n}{ }^{(A)}$ is now given by a solution of Eq. (35), with $v_{A}(R)$ replaced by (39a), which behaves as a plane wave plus outgoing spherical wave for large internuclear separations. It is, in atomic units,

$$
\begin{aligned}
\chi_{n}{ }^{(A)}(R)= & \left(\frac{\pi}{2 k R}\right)^{\frac{1}{2}} \sum_{l}(2 l+1) i^{l} A_{l}{ }^{(+)} \\
& \times\left[J_{l+\frac{1}{2}}(k R)-a_{l} J_{-l-\frac{1}{2}}(k R)\right] P_{l}(\cos \theta),
\end{aligned}
$$

${ }^{10}$ S. C. Wang, Phys. Rev. 31, 579 (1928). 


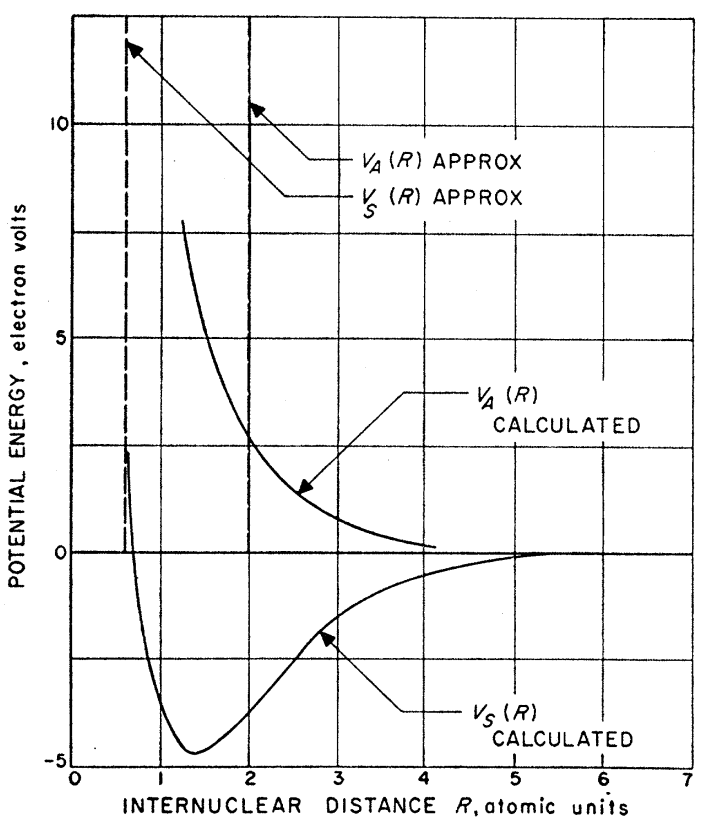

Frg. 1. The interaction potential between two hydrogen atoms. The solid lines represent the actual potential energies as calculated by A. S. Collidge and H. M. James [J. Chem. Phys. 1, 825 (1933); 6, $730(1938)]$.

where $J$ is the Bessel function and

$A_{l}{ }^{(+)}=\left[1+(-1)^{l} i a_{l}\right]^{-1}, \quad a_{l}=J_{l+\frac{1}{2}}(2 k) / J_{-l-\frac{1}{2}}(2 k)$.

The summation runs over all even integers if $n=3$ (symmetric case) and it runs over all odd integers if $n=1$ (antisymmetric case).

The wave function $\chi_{n}{ }^{(S)}$ for the final state, on the other hand, is a solution of Eq. (37), with $v_{S}(R)$ replaced by $(39 \mathrm{~b})$ which behaves as a plane wave plus incoming spherical wave for large internuclear separations. ${ }^{11}$ It is, again in atomic units,

$\chi_{n}{ }^{(S)}=\left(\frac{\pi}{2 k R}\right)^{\frac{1}{2}} \sum_{l}(2 l+1) i^{l} A_{l}{ }^{(-)}$

where

$$
\times\left[J_{l+\frac{1}{2}}(k R)-a_{l} J_{-l-\frac{1}{2}}(k R)\right] P_{l}(\cos \alpha),
$$

$$
\begin{aligned}
A_{l}{ }^{\prime(-)} & =\left[1-(-1)^{l} i a_{l}\right]^{-1}, \\
a_{l}{ }^{\prime} & =J_{l+\frac{1}{2}}(0.6 k) / J_{-l-\frac{1}{2}}(0.6 k),
\end{aligned}
$$

and

$$
\cos \alpha=\cos \theta \cos \theta^{\prime}+\sin \theta \sin \theta^{\prime} \cos \left(\phi-\phi^{\prime}\right) .
$$

Here again the summation runs over even (odd) integers if the wave function is symmetric, $n=1$ (antisymmetric, $n=3)$. The polar angles $\theta^{\prime}, \phi^{\prime}$ in (41b) define the direction of the scattered wave relative to the incoming wave. For low velocities it is sufficient to

11 The use of incoming rather than outgoing spherical waves as a boundary condition for the final state can be shown to be correct under the assumption that the final state contains only a plane wave at $t=+\infty$ [G. Breit and H. A. Bethe, Phys. Rev. 93, 888 (1954)]. take only the first partial wave of the expansions (40) and (41) so that we have the choice of two different initial and two different final nuclear wave functions depending on the symmetry involved. They are, for the initial state,

$$
\begin{aligned}
& \chi_{S^{(A)}}=(k R)^{-1} e^{-2 i k} \sin k(R-2), \\
& \chi_{A}{ }^{(A)}=\frac{-3 i}{1-i a_{1}}\left(\frac{\pi}{2 k R}\right)^{\frac{1}{2}}\left[J_{\frac{3}{2}}(k R)-a_{1} J_{-\frac{3}{2}}(k R)\right] \cos \theta
\end{aligned}
$$

with

$$
a_{1}=J_{\frac{3}{2}}(2 k) / J_{-\frac{3}{2}}(2 k),
$$

and for the final state

$\chi_{S}{ }^{(S)}=(k R)^{-1} e^{+0.6 i k} \sin k(R-0.6)$,

$\chi_{A}{ }^{(S)}=\frac{-3 i}{1+i a_{1}}\left(\frac{\pi}{2 k R}\right)^{\frac{1}{2}}\left[J_{\frac{3}{2}}(k R)-a_{1}{ }^{\prime} J_{-\frac{3}{2}}(k R)\right] \cos \alpha$,

with

$$
a_{1}^{\prime}=J_{\frac{3}{3}}(0.6 k) / J_{-\frac{3}{2}}(0.6 k) .
$$

Collecting all results, we see that the wave function for the initial state is given by Eq. (34) together with Eqs. (38) and (42), whereas the wave function of the final state is given by Eq. (36) together with Eqs. (38) and (43).

\section{b. Determination of the Matrix Element}

In evaluating the matrix element responsible for the transition between the states specified in the preceding section due to the interaction (31), considerable simplifications arise from the inherent symmetry of the problem. First of all we notice that there are no contributions from the orbit-orbit interaction $V_{1}$ of $\mathrm{Eq}$. (31a) since $V_{1}$ does not contain any spin interaction and the transition considered changes the multiplicity of the states involved. Furthermore the interaction operator $B_{12}$ representing the coupling between the two electrons also does not give any contributions to the matrix element as can readily be seen from symmetry considerations. Hence our task is reduced to the evaluation of the matrix elements of the following operator:

$$
\begin{aligned}
V & =V_{\mathrm{so}}+V_{\mathrm{ss}}, \\
V_{\mathrm{so}} & =V_{2}(A, 1)+V_{2}(A, 2)+V_{2}(B, 1)+V_{2}(B, 2), \\
V_{\mathrm{ss}} & =V_{3}(A, 1)+V_{3}(A, 2)+V_{3}(B, 1)+V_{3}(B, 2) .
\end{aligned}
$$

The argument indicates the various particles involved. $V_{\text {so }}$ represents the spin-orbit and $V_{\mathrm{ss}}$ the spin-spin interaction [Eqs. (31b), (31c), respectively]. A straightforward application of the theorem of conservation of angular momentum [the interaction (31) commutes with both the total angular momentum and its $z$ component] reveals then that the spin-orbit coupling $V_{\text {so }}$ possesses only two nonvanishing matrix elements in our approximation which consists of neglecting all components of the nuclear wave functions with angular 
momentum $l \geqslant 2$. The 2 possible transitions are

$$
S_{1}{ }^{1}(A, B)\left\{\begin{array}{c}
S_{1}^{3}(1,2) \\
S_{2}{ }^{3}(1,2)
\end{array}\right\} \rightarrow S_{1}^{1}(A, B) S_{1}^{1}(1,2) .
$$

The notation has been defined in Eq. (33). The two transitions (45) are accompanied by a change of the orbital angular momentum from $l=0$ to $l=+1$. The spin-spin interaction $V_{\mathrm{ss}}$, on the other hand, gives rise to the following possible transitions between any of the 12 triplet states, Eq. (34), and any of the 4 singlet states, Eq. (36) :

$$
\begin{aligned}
& \left.\begin{array}{l}
S_{1}{ }^{1}(A, B) \\
S_{3}{ }^{3}(A, B)
\end{array}\right\} \times S_{3}{ }^{3}(1,2) \rightarrow\left\{\begin{array}{c}
S_{3}{ }^{3}(A, B) \\
S_{1}{ }^{1}(A, B)
\end{array}\right\} \times S_{1}{ }^{1}(1,2), \\
& \left.\begin{array}{l}
S_{1}{ }^{1}(A, B) \\
S_{2}{ }^{3}(A, B)
\end{array}\right\} \times S_{1}{ }^{3}(1,2) \rightarrow\left\{\begin{array}{c}
S_{1}{ }^{3}(A, B) \\
S_{1}{ }^{1}(A, B)
\end{array}\right\} \times S_{1}{ }^{1}(1,2), \\
& \left.\begin{array}{l}
S_{1}{ }^{1}(A, B) \\
S_{1}{ }^{3}(A, B)
\end{array}\right\} \times S_{2}{ }^{3}(1,2) \rightarrow\left\{\begin{array}{c}
S_{2}{ }^{3}(A, B) \\
S_{1}{ }^{1}(\mathrm{~A}, B)
\end{array}\right\} \times S_{1}{ }^{1}(1,2) .
\end{aligned}
$$

The actual evaluation of the matrix elements between the spin states specified by Eqs. (45) and (46) proceeds then in a straightforward manner. After some calculation it is found that the operator $V_{\text {so }}$ leads to the following matrix elements for the two transitions (45):

$$
\begin{array}{r}
M_{ \pm}=\frac{\hbar^{2} e^{2}}{\sqrt{2}\left(2 \pi m M c^{2} a_{0}{ }^{3}\right)} \int \chi_{A}(S)(R)^{*} l(R) \\
\times \mathscr{L}_{ \pm} \chi_{S}{ }^{(A)}(R) d^{3} R,
\end{array}
$$

with

$$
\begin{aligned}
& l(R)=\frac{1}{2 \pi} \int d^{3} r \frac{\cos \theta}{r^{2}} e^{-2|\mathbf{r}-\mathbf{R}|} \\
& =\frac{1}{2 R^{2}}\left[1-\left(1+2 R+2 R^{2}\right) e^{-2 R}\right],
\end{aligned}
$$

$$
\mathfrak{L}_{ \pm}=\partial / \partial x_{A} \pm i \partial / \partial y_{A}
$$

The nuclear wave functions are specified by Eqs. (42) and (43). $M_{+}$corresponds to the first and $M_{-}$to the second transition (45). The factor $a_{0}^{-3} \quad\left(a_{0}=\right.$ Bohr's radius) is due to the use of atomic units. The operator $V_{\text {ss, }}$, on the other hand, gives rise to the following matrix elements: Transition (46a) leads to

$$
M_{1}=\frac{\hbar^{2} e^{2}}{2 \pi m M c^{2} a_{0}^{3}} \int \chi_{A}^{(S)}(R)^{*} f_{1}(R) \chi_{A}^{(A)}(R) d^{3} R .
$$

Transition (46b) leads to

$M_{1}^{\prime}=\frac{\hbar^{2} e^{2}}{2 \pi m M c^{2} a_{0}{ }^{3}} \int \chi_{S^{(S)}}(R)^{*} f_{1}(R) \chi_{S}{ }^{(A)}(R) d^{3} R$,

with

$$
f_{1}(R)=\frac{2}{3} e^{-2 R}-\frac{1}{4 \pi} \int d^{3} r \frac{1-3 \cos ^{2} \theta}{r^{3}} e^{-2|\mathrm{r}-\mathrm{R}|}
$$

Transitions (46c) and (46e) lead to

$$
M_{2}=\frac{\hbar^{2} e^{2}}{2 \pi m M c^{2} a_{0}^{3}} \int \chi_{A}^{(S)}(R)^{*} f_{2}(R) \chi_{A}^{(A)}(R) d^{3} R .
$$

Transitions (46d) and (46f) lead to

$$
M_{2}^{\prime}=\frac{\hbar^{2} e^{2}}{2 \pi m M c^{2} a_{0}^{3}} \int \chi_{S}^{(S)}(R)^{*} f_{2}(R) \chi_{S}{ }^{(A)}(R) d^{3} R,
$$

with

$$
f_{2}(R)=\frac{2}{3} e^{-2 R}-\frac{1}{4 \pi} \int d^{3} r \frac{1-\frac{3}{2} \sin ^{2} \theta}{r^{3}} e^{-2|\mathrm{r}-\mathrm{R}|} .
$$

The integrals over $\mathbf{r}$ defining $f_{1}(R)$ and $f_{2}(R)$ are singular. They can easily be made convergent by excluding from the integration a small sphere with center at the origin and radius $\epsilon$ and taking the limit $\epsilon=0$ after having performed the integration. ${ }^{3}$ This integration is sketched in Appendix I. The result is

$$
\begin{aligned}
& f_{1}(R)=\frac{2}{3} e^{-2 R}+\frac{1}{2 R^{3}}\left[1-\left(1+2 R+2 R^{2}\right) e^{-2 R}\right] \\
& f_{2}(R)=\frac{2}{3} e^{-2 R}-\frac{1}{4 R^{3}}\left[1-\left(1+2 R+2 R^{2}\right) e^{-2 R}\right] .
\end{aligned}
$$

The further evaluation of the matrix elements is greatly simplified by the fact that the initial nuclear wave function $\chi^{(A)}$ is zero for $R<2$ (the strong repulsion does not allow the particles to approach each other too much). We are therefore able to neglect all terms containing the exponential $e^{-2 R}$ to a good approximation. Inserting the expressions (42) and (43) for the nuclear wave functions into the matrix elements (47), (50), (51), (53), and (54), and performing the angular integration, leads (with the abbreviation $A=\pi e^{2} \hbar^{2}$ / $\left.2 m M c^{2} a_{0}^{3}\right)$ to

$$
\begin{aligned}
& M_{ \pm}=\frac{1}{4} A L(k) \sin \theta^{\prime} e^{ \pm i \phi^{\prime}}, \\
& M_{1}=2 M_{2}=A F(k) \cos \theta^{\prime}, \\
& M_{1}^{\prime}=2 M_{2}^{\prime}=A G(k),
\end{aligned}
$$

where

$$
\begin{array}{r}
L(k)=\frac{e^{-2 i k}}{1-i a_{1}{ }^{\prime}} \int_{2 k}^{\infty}(\pi u)^{-\frac{1}{2}}\left[J_{\frac{3}{2}}(u)-{\left.a_{1}{ }^{\prime} J_{-\frac{3}{2}}(u)\right]}\right. \\
\times d\left(\frac{\sin (u-2 k)}{u}\right),
\end{array}
$$

$$
\begin{array}{r}
F(k)=\frac{3}{\left(1-i a_{1}\right)\left(1-i a_{1}^{\prime}\right)} \int_{2 k}^{\infty}\left[J_{\frac{3}{2}}(u)-a_{1} J_{-\frac{3}{2}}(u)\right] \\
\quad \times\left[J_{\frac{3}{2}}(u)-a_{1}^{\prime} J_{-\frac{3}{2}}(u)\right] \frac{d u}{u^{2}} \\
G(k)=2 e^{-2.6 i k} \int_{2 k}^{\infty} \sin (u-2 k) \sin (u-0.6 k) \frac{d u}{u^{3}},
\end{array}
$$


$k=M v a_{0} / \sqrt{2} \hbar$ is the wave vector in atomic units and $\theta^{\prime}, \phi^{\prime}$ are the polar angles defining the direction of the scattered wave with respect to the incident wave.

We are now in a position to actually compute the cross section of a spin-flip reaction for a polarized beam of atomic hydrogen. Since we are dealing with unpolarized protons we have to average over all possible initial proton spin states and to sum over all final ones. The total collision cross section is then obtained in the usual manner by evaluating the transition probability according to Eq. (30), integrating over all possible scattering angles $\theta^{\prime}, \phi^{\prime}$ and dividing by the current density of the incoming beam $j=\hbar k / M$ (normalized to unit volume). It is found after some calculation that for an electronic transition $S_{3}{ }^{3}(1,2) \rightarrow S_{1}{ }^{1}(1,2)$ the total spinflip cross section is

$$
Q_{1}=\frac{\pi}{16} r_{0}^{2}\left\{\frac{1}{3}|F(k)|^{2}+|G(k)|^{2}\right\},
$$

whereas for the electronic transition $S_{1}{ }^{3}(1,2) \rightarrow S_{1}{ }^{1}(1,2)$ and $S_{2}{ }^{3}(1,2) \rightarrow S_{1}{ }^{1}(1,2)$ the cross section is

$$
Q_{2}=\frac{\pi}{8} r_{0}^{2}\left\{\frac{1}{3}|L(k)|^{2}+\frac{2}{3}|F(k)|^{2}+2|G(k)|^{2}\right\} .
$$

In formulas (64) and (65), $r_{0}=e^{2} / 4 \pi m c^{2}$ is the classical radius of the electron. In order to evaluate the 3 functions $L, F$, and $G$, we note that $2 k=0.244 \sqrt{ } T$, where $T$ is the temperature in ${ }^{\circ} \mathrm{K}$ of the colliding particles so that for small $T$ the integrals (61), (62), and (63) which define $L, F$, and $G$ may be expanded into a power series in $2 k$. The result of the somewhat lengthy calculations is listed in Appendix II.

To obtain the final result, namely the cross section for scattering of two polarized beams of hydrogen atoms accompanied by spin flip, we still have to perform an averaging process. For, let us suppose that the two crossed beams of $\mathrm{H}$ atoms are polarized in a spacefixed $Z$ direction. The electronic spin function may then be represented by

$$
S(1,2)=\alpha_{1}(Z) \alpha_{2}(Z),
$$

where $\alpha(Z)$ is the "spin up" wave function for the $Z$ direction, defined in exactly the same way as the "spin up" function $\alpha$ we used, which was associated with the direction of relative motion $\mathbf{R}$. The spin function (66) can easily be expressed as a linear combination of the 3 symmetric spin functions used previously. Denoting the polar angles which determine the direction of relative motion $R$ with respect to the $Z$ direction by $\theta_{S}$ and $\phi_{S}$, it is with

$$
S(1,2)=\alpha_{1} S_{1}{ }^{3}(1,2)+\alpha_{2} S_{2}{ }^{3}(1,2)+\alpha_{3} S_{3}{ }^{3}(1,2),
$$

$$
\begin{aligned}
& \alpha_{1}=\cos ^{2}\left(\theta_{S} / 2\right), \\
& \alpha_{2}=e^{-2 i \phi_{S}} \sin ^{2}\left(\theta_{S} / 2\right), \\
& \alpha_{3}=\sqrt{2} \cos \left(\theta_{S} / 2\right) \sin \left(\theta_{S} / 2\right) .
\end{aligned}
$$

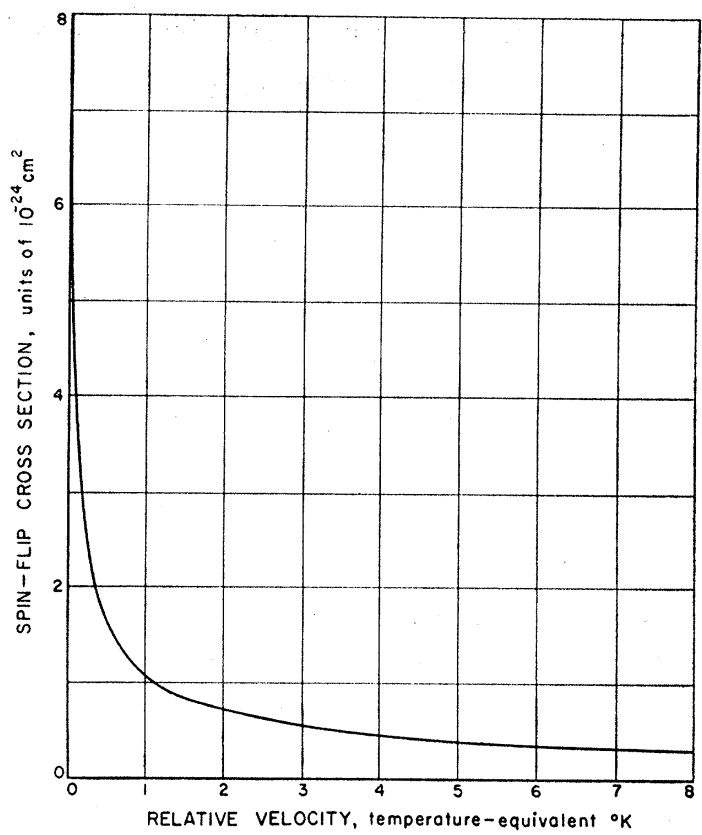

FIG. 2. The spin-flip cross section $Q$ as a function of relative velocity.

It follows immediately from (64), (65), and (68) that the total cross section for spin flip is given by

$$
Q=\left(\left|\alpha_{1}\right|^{2}+\left|\alpha_{2}\right|^{2}\right) Q_{2}+\left|\alpha_{3}\right|^{2} Q_{1} .
$$

In the special case $\theta_{S}=\pi / 2$ (polarization perpendicular to the motion), we have from (69)

$$
Q=\frac{1}{2}\left(Q_{1}+Q_{2}\right) \text {. }
$$

Observing Eqs. (64) and (65), we get in the special case of perpendicular polarization

$$
Q=\frac{\pi}{16} r_{0}^{2}\left\{\frac{1}{3}|L(k)|^{2}+\frac{5}{6}|F(k)|^{2}+\frac{5}{2}|G(k)|^{2}\right\} .
$$

From Eq. (A4) of Appendix II we see that the lowvelocity limit is given by

$$
\lim _{k=0} Q=\frac{5 \pi}{8} r_{0}^{2}[\ln (4 \gamma k)]^{2}
$$

exhibiting a logarithmic divergence. Finally it has to be borne in mind that the cross section, Eq. (71), has been calculated with the assumption of a gyromagnetic ratio $g_{p}=2$ for the proton (Dirac equation). Actually the observed value is $g_{p}=5.59$, so that we merely have to multiply Eq. (71) by $(5.59 / 2)^{2}=7.82$ in order to correct for this fact. Figure 2 shows the cross section $Q$ as a function of the relative velocity of the colliding particles. There it is seen that $Q$ is of the order of $10^{-24}$ $\mathrm{cm}^{2}$ (around $1^{\circ} \mathrm{K}$ ) so that the probability of a spin flip per collision is of the order of

$$
P=Q / Q_{\mathrm{el}}=10^{-10}
$$


where we took for $Q_{\mathrm{el}}$ the elastic scattering cross section

$$
Q_{\mathrm{el}}=4 \pi\left(2.5 \times 10^{-8}\right)^{2} \mathrm{~cm}^{2}
$$

assuming that the classical cross section corresponds to a diameter of $2.5 \mathrm{~A}$.

\section{APPENDIX I}

The integrals occurring in formulas (52) and (55),

and

$$
I_{1}=\frac{1}{4 \pi} \int d^{3} r \frac{1-3 \cos ^{2} \theta}{r^{3}} e^{-2|\mathbf{r}-\mathbf{R}|}
$$

$$
I_{2}=\frac{1}{4 \pi} \int d^{3} r \frac{1-\frac{3}{2} \sin ^{2} \theta}{r^{3}} e^{-2|\mathbf{r}-\mathbf{R}|},
$$

can easily be evaluated by substitution. Since $\theta$ is the angle between $\mathbf{r}$ and $\mathbf{R}$, the substitution

leads to

$$
|\mathbf{r}-\mathbf{R}|=+\left(r^{2}+R^{2}-2 r R \cos \theta\right)^{\frac{1}{2}}=u
$$

$$
\begin{aligned}
& I_{1}=\frac{1}{2 R} \lim _{\epsilon=0} \int_{\epsilon}^{\infty} \frac{d r}{r^{2}} \int_{|r-R|}^{r+R} u d u \\
& \quad \times e^{-2 u}\left[1-\frac{3}{4 R^{2} r^{2}}\left(r^{2}+R^{2}-u^{2}\right)\right], \\
& I_{2}=\frac{1}{2 R} \lim _{\epsilon=0} \int_{\epsilon}^{\infty} \frac{d r}{r^{2}} \int_{|r-R|}^{r+R} u d u \\
& \quad \times e^{-2 u}\left[-\frac{1}{2}+\frac{3}{8 R^{2} r^{2}}\left(r^{2}+R^{2}-u^{2}\right)\right] .
\end{aligned}
$$

In order to perform first the much simpler $r$ integration, both $I_{1}$ and $I_{2}$ are converted into

$$
\begin{aligned}
I_{1}= & \frac{1}{2 R} \lim _{\epsilon=0}\left\{\int_{0}^{R-\epsilon} u d u\right. \\
& \times e^{-2 u} \int_{R-u}^{R+u} \frac{d r}{r^{2}}\left[1-\frac{3}{4 R^{2} r^{2}}\left(r^{2}+R^{2}-u^{2}\right)\right] \\
& \left.+\int_{R+\epsilon}^{\infty} u d u e^{-2 u} \int_{u-R}^{u+R} \frac{d r}{r^{2}}\left[1-\frac{3}{4 R^{2} r^{2}}\left(r^{2}+R^{2}-u^{2}\right)\right]\right\},
\end{aligned}
$$

and an analogous expression for $I_{2}$. The second double integral of the last expression vanishes (the same holds true for the corresponding expression for $I_{2}$ ), whereas the first yields after simple calculation the result Eq. (56) of the text.

\section{APPENDIX II}

The integrals (61), (62), and (63) defining the functions $L, F$, and $G$ may readily be evaluated by successive partial integration. For small values of $k$ the following expansions hold $(\epsilon=2 k)$ :

$$
\begin{aligned}
|L(k)|^{2}= & \frac{1}{18}\left\{1-\frac{2}{\pi}[8+\ln (2 \gamma \epsilon)] \epsilon\right. \\
& +\left[\frac{64}{\pi^{2}}-1+\frac{32}{\pi^{2}}(2 \gamma \epsilon)+-\frac{4}{\pi}[\ln (2 \gamma \epsilon)]^{2}\right] \epsilon^{2} \\
& +\frac{1}{9 \pi}[70+4 \ln (2 \gamma \epsilon)] \epsilon^{3}+\left[\frac{19}{60}+\frac{0.81}{\pi^{2}} \ln (2 \gamma \epsilon)\right. \\
|F(k)|^{2}= & \left.\left.\frac{9}{4}\left(1-\frac{6}{5} \epsilon^{2}+\frac{8}{7} \epsilon^{4}\right), \quad \frac{2}{3 \pi^{2}}[\ln (2 \gamma \epsilon)]^{2}\right] \epsilon^{4}\right\}, \quad, \\
\text { whereas } & |G(k)|^{2} \text { is given by } \\
|G(k)|^{2}= & {\left[\pi \sin 1.3 \epsilon+\frac{\sin 0.7 \epsilon}{\epsilon}-2 \cos 1.3 \epsilon \operatorname{Ci}(2 \epsilon)\right.}
\end{aligned}
$$

valid for all values of $\epsilon=2 k$. In the above formulas, $\gamma=1.78$ is the Euler constant, $\mathrm{Ci}(x)$ and $\mathrm{Si}(x)$ are the cosine integral and the sine integral, respectively, defined by

$$
\operatorname{Si}(x)=\int_{0}^{x} \frac{\sin t}{t} d t, \quad \operatorname{Ci}(x)=-\int_{x}^{\infty} \frac{\cos t}{t} d t .
$$

The low-velocity limit of (A1), (A2), and (A3) is given by

$$
\begin{aligned}
& \lim _{k=0}|L(k)|^{2}=1 / 18, \\
& \lim _{k=0}|F(k)|^{2}=9 / 4, \\
& \lim _{k=0}|G(k)|^{2} \sim[2 \ln (4 \gamma k)]^{2} .
\end{aligned}
$$

